\title{
Correlatos psicosociales de depresión y riesgo de suicidio en trabajadoras sexuales del Estado de Hidalgo, México
}

\author{
Catalina González-Forteza, ' Eva María Rodríguez,' Patricia Fuentes de lturbe, ' \\ Leticia Vega,' Alberto Jiménez Tapia'
}

Artículo original

\section{SUMMARY}

Available data on mental health among female sex workers in Mexico are scarce. The scarce evidence shows that the prevalence of depression and suicidal problems is much higher than in the general population. The objective of this article was to explore the psychosocial factors associated with depression and suicide risk in a sample of 103 sex workers from the state of Hidalgo, Mexico. Among them, the frequency of depression and suicide risk was higher than for women in the general population $(39.8 \%$ and $3.0 \%$, respectively). The variables that predicted these illnesses were a bad relationship with the mother, the negative health effects due to alcohol use, partner violence, and sexual violence. It is necessary to consider these results to promote actions that reduce or eliminate partner violence, promote the acquisition of skills to manage the negative consequences of violence and implement strategies to reduce the harm caused by alcohol consumption in this vulnerable group of the population.

Key words: Depression, sex workers, suicide risk, alcohol use.

\section{RESUMEN}

Los datos disponibles sobre la salud mental en trabajadoras sexuales en México son escasos. Sin embargo la poca evidencia disponible muestra que las prevalencias de depresión y problemática suicida son mucho más elevadas que en la población general. El objetivo de este artículo es explorar los factores psicosociales que se relacionan con la depresión y el riesgo de suicidio en una muestra de 103 trabajadoras sexuales del Estado de Hidalgo, México. Los resultados muestran que las frecuencias de depresión y riesgo de suicidio fueron más elevadas que en las mujeres de la población general 39.8 y $3.0 \%$, respectivamente). Las variables que predijeron estos malestares fueron la mala relación con la madre, los efectos perjudiciales del consumo de alcohol sobre la salud física y las actividades domésticas, la violencia de la pareja y la violencia sexual. Es necesario considerar estos resultados para propiciar acciones que reduzcan o eliminen la violencia de pareja, fomenten la adquisición de habilidades para el manejo de las consecuencias negativas de la violencia, así como implementar estrategias para reducir el daño ocasionado por el consumo de alcohol en este grupo poblacional en situación de vulnerabilidad.

Palabras clave: Depresión, riesgo suicida, trabajadoras sexuales, consumo de alcohol.

\section{INTRODUCCIÓN}

La depresión y la problemática suicida se encuentran entre los principales problemas de salud mental pública que enfrenta la población de México. En la Encuesta Nacional de Epidemiología Psiquiátrica, que se realiza con población general entre los 18 y 65 años de edad, se encontró una prevalencia de problemas depresivos alguna vez en la vida de $4.8 \%$ ( $2.6 \%$ en hombres y $6.8 \%$ en mujeres). ${ }^{1}$ Se ha documentado que en $2.0 \%$ de la población, éstos se inician durante la infancia o la adolescencia, que no reciben tratamiento y que están relacionados de manera directa con la problemática suicida. ${ }^{2,3}$
El suicidio en México es un fenómeno que ha aumentado de manera constante en los últimos años. En 1990 se registraron 1941 muertes por suicidio $(2.4 \times 100000$ habitantes), 284 fueron mujeres (0.69 x 100000); y para el año 2012 la cifra aumentó a 5550 ( 5 x 100000 habitantes), de los cuales 1077 fueron mujeres $(2 \times 100000)$. Los datos colocan al suicidio entre las veinte principales causas de muerte y la tercera entre las defunciones accidentales y violentas en las mujeres. ${ }^{4}$ Dada la magnitud del fenómeno, es necesario el estudio de las conductas que se incluyen en la problemática suicida -ideación, planeación e intento- ${ }^{5}$ y más tratándose del intento de suicidio que es uno de los principales factores de riesgo del suicidio consumado. ${ }^{6}$

Dirección de Investigaciones Epidemiológicas y Psicosociales. Instituto Nacional de Psiquiatría Ramón de la Fuente Muñiz.

Correspondencia: Alberto Jiménez Tapia. Dirección de Investigaciones Epidemiológicas y Psicosociales, Instituto Nacional de Psiquiatría Ramón de la Fuente Muñiz. Calz. México-Xochimilco 101, San Lorenzo Huipulco, Tlapan, 14370 México, D.F. Tel: (55) 4160 - 5179. E-mail: alberj@imp.edu.mx 
El interés por el estudio de diferentes factores relacionados con la salud mental en la población se ha mantenido en los años recientes; sin embargo, la evidencia es aún limitada cuando se trata de poblaciones ocultas o en situación de vulnerabilidad, como es el caso de las trabajadoras sexuales, en quienes ha sido más frecuente el estudio de temas relacionados con la salud sexual, las infecciones de transmisión sexual y el VIH; y aunque existen menos estudios sobre sus condiciones de salud mental los datos en la literatura científica muestran estimaciones en las que este grupo tiene una frecuencia de depresión de moderada a grave mayor que las mujeres de la población general. ${ }^{7}$

Asimismo, la información disponible sobre la problemática suicida en trabajadoras sexuales es escasa, pero se sabe que su frecuencia de intento de suicidio alguna vez en la vida es muy elevada $(53 \%)^{8}$ y mayor que la proporción de la población general femenina. También se ha visto que en este grupo el suicidio es una causa de muerte importante $(4.5 \%)^{9}$ y que la problemática suicida es un fenómeno con una presencia considerable; diferentes estudios han reportado que la ideación llega al 14\% y el intento reciente oscila entre 8 y $19 \% .^{10,11}$

Se han identificado diferentes correlatos asociados con la presencia de problemática suicida y depresión en mujeres que ejercen el trabajo sexual; estos factores suelen formar parte de los contextos en los que ellas desarrollan su actividad y que, en ocasiones, son inherentes a la misma. Se sabe que existen condiciones de violencia por parte de sus parejas y de sus clientes, abuso de alcohol, consumo de otras drogas y una salud mental disminuida, lo que incluye tener baja autoestima, problemas depresivos y ansiedad. ${ }^{10,11}$

El trabajo sexual tiene una relevancia particular y ciertas características que lo convierten en un fenómeno complejo. El estudio de algunos problemas de salud mental y sus determinantes psicosociales se ha abarcado de manera limitada en las mujeres que ejercen dicha actividad. Este trabajo se plantea como una contribución que tiene el objetivo de explorar los factores psicosociales relacionados con la depresión y la problemática suicida en una muestra de trabajadoras sexuales del Estado de Hidalgo, México.

\section{MÉTODO}

Se realizó un estudio transversal descriptivo en el Estado de Hidalgo. La muestra fue no probabilística e incluyó a 103 trabajadoras sexuales. El criterio de selección fue que las participantes ejercieran el trabajo sexual, que tuvieran entre 18 y 65 años de edad y que supieran leer y escribir.

\section{Instrumento}

Se utilizó un instrumento diseñado ex profeso para este estudio para realizar las entrevistas de manera semiestructurada con base en las 287 preguntas para explorar diferentes áreas relacionadas con la salud de esta población; en este trabajo se reportan datos sociodemográficos, historia escolar, depresión, riesgo de suicidio y consumo de alcohol. Las variables dependientes fueron: depresión y riesgo de suicidio, las cuales se evaluaron con el MINI (International Neuropsychiatric Interview 5.0.0). ${ }^{12}$

\section{Procedimiento}

El trabajo de campo se llevó a cabo en el año 2010 en una Jurisdicción Sanitaria del estado de Hidalgo, donde las mujeres acudían a revisión médica para que se les otorgara el permiso y pudieran trabajar ese fin de semana.

Las entrevistas las realizó un equipo de tres psicólogos capacitados previamente para la aplicación del instrumento y éstas se aplicaron en formato cara a cara; con una duración promedio de dos a tres horas, en cubículos separados para mantener la privacidad durante las entrevistas.

\section{Consideraciones éticas}

El Comité de Ética del Instituto Nacional de Psiquiatría Ramón de la Fuente Muñiz aprobó el proyecto para su realización. Las entrevistas se efectuaron previo consentimiento informado de las mujeres y con el pleno conocimiento de los objetivos de la investigación, asegurándoles la absoluta confidencialidad en el manejo de la información. La participación fue voluntaria y se les indicó que podían abandonar el estudio en el momento que así lo desearan; no hubo rechazos.

\section{Análisis estadísticos}

Se realizaron análisis de frecuencias para describir las características sociodemográficas de la muestra; se calificaron las escalas y se recodificaron los puntajes para construir grupos de caso y de no caso de depresión (D) y riesgo de suicidio (RS), de acuerdo con los criterios del MINI. Finalmente, se realizó un análisis de árboles de detección automática de interacciones con la técnica $\mathrm{CHAID}^{13}$ para cada condición con las variables independientes agrupadas en segmentos: familia (estilos de crianza recibidos y relación con los padres), pareja (duración con la pareja actual, violencia -emocional, física y sexual- y miedo a la pareja), consumo de alcohol (efectos perjudiciales y consecuencias negativas), consumo de drogas en los últimos 12 meses, violencia sexual (abuso y violación alguna vez en la vida), esclavitud sexual, violencia en el trabajo, e impulsividad.

\section{RESULTADOS}

La edad de las participantes fluctuó entre los 18 y 47 años de edad. La mayoría de las participantes (67\%) eran de distintos Estados del país, incluido Hidalgo; $31 \%$ de la Ciudad de 
México y 2\% extranjeras. El 70\% de la muestra tenía estudios de secundaria o menos. Cerca de la mitad de las mujeres eran casadas y la mayoría tenía entre uno y cinco años ejerciendo el trabajo sexual. El 80\% de ellas reportó un consumo elevado de alcohol y la mitad ejercía el trabajo sexual únicamente en bares (cuadro 1).

La clasificación de casos de depresión y riesgo de suicidio mostró que $40 \%$ de las mujeres tienen problemática depresiva o riesgo de suicidio cuando se considera cada una de manera independiente y que $25 \%$ de las mujeres de la muestra presentan ambas problemáticas de manera simultánea (cuadro 2).

Los análisis de árboles de decisión generaron diferentes modelos con las dos variables dependientes: casos de depresión -D- y casos de riesgo de suicidio -RS-.

Cuadro 1. Características de las trabajadoras sexuales de Hidalgo $(n=103)$

Edad (media $=28.8, \mathrm{DE}=6.7$ )

- 18-24 años

- 25-34 años

- 35-44 años

- >45

Escolaridad

- Sin escolaridad

- Primaria o inferior

- Secundaria

- Bachillerato

- Licenciatura inconclusa

- Licenciatura terminada

Estado civil

- Soltera

49.0

- Casada/unión libre

28.0

- Divorciada/separada

- Viuda

14.0

Tiempo en el trabajo sexual

- $<1$ año

- 1-5 años

- >5 años

56.3

Patrón consumo alcohol

- Elevado

- Moderado

78.0

- Leve

- Infrecuente

14.0

6.0

2.0

Trabajo sexual y otras actividades*

- Sólo trabajo sexual en bares

48.0

- Sólo trabajo sexual en la calle

16.0

- Personal de limpieza

25.0

- Empleada

14.0

- Otras actividades

9.0

*Las categorías no son mutuamente excluyentes.
Cuadro 2. Distribución de casos de depresión y de riesgo de suicidio en trabajadoras sexuales de Hidalgo $(n=103)$

\begin{tabular}{lcc}
\hline Tipo de condición & $\mathrm{F}$ & $\%$ \\
\hline Depresión & 41 & 39.8 \\
Sin depresión & 62 & 60.2 \\
Riesgo de suicidio & 40 & 38.8 \\
Sin riesgo de suicidio & 63 & 61.2 \\
Presencia de ambas condiciones & 26 & 25.2 \\
\hline
\end{tabular}

\section{Casos de depresión}

El modelo generó dos segmentos en el análisis con el área de familia respecto a la relación que la madre tuvo con su hija. Cuando ésta fue de mala a muy mala se predijo $75 \%$ de los casos de depresión y cuando la relación fue buena, se predijo a $62 \%$ de quienes no presentaron depresión. En el cuadro 3 se muestran los porcentajes de predicción correcta; en este caso, el modelo tuvo una especificidad de $70 \%$ y una sensibilidad de $67 \%$.

En el análisis del área de la pareja, el modelo generó dos segmentos finales: el maltrato emocional en alguna ocasión por parte de la pareja, predijo $64 \%$ de los casos de depresión y cuando no lo habían sufrido, se predijo 71\% de no casos de depresión (especificidad=34\%, sensibilidad=90\%) (cuadro 3).

El modelo con violencia sexual también generó dos segmentos finales que predijeron la presencia de depresión en $74 \%$ de los casos de depresión cuando ellas reportaron haber sido víctimas de abuso sexual alguna vez en su vida, y 51\% de los no casos de depresión cuando no fueron víctimas de abuso sexual (especificidad $=82 \%$, sensibilidad $=40 \%$ ). (cuadro 3 )

El resto de las variables independientes que se incluyeron en los análisis no generaron modelos de clasificación.

\section{Riesgo de suicidio}

El modelo resultante generó dos segmentos finales con respecto al área de la familia; éstos predijeron $56 \%$ de los casos

Cuadro 3. Porcentajes de clasificación de casos y no casos de depresión en trabajadoras sexuales de Hidalgo ( $n=103)$

\begin{tabular}{lccc}
\hline & \multicolumn{3}{c}{ Pronosticado } \\
\cline { 2 - 4 } & $\begin{array}{c}\text { Sin } \\
\text { depresión (\%) }\end{array}$ & $\begin{array}{c}\text { Depresión } \\
(\%)\end{array}$ & $\begin{array}{c}\text { Correcto } \\
(\%)\end{array}$ \\
\hline Familia: Relación con la madre & & & \\
Sin depresión & 31.0 & 13.0 & 70.5 \\
Depresión & 19.0 & 39.0 & 67.2 \\
Pareja: Maltrato emocional & & & \\
Sin depresión & 15.0 & 29.0 & 34.1 \\
Depresión & 6.0 & 52.0 & 89.7 \\
Violencia sexual: Abuso sexual & & & \\
Sin depresión & 37.0 & 8.0 & 82.2 \\
Depresión & 35.0 & 23.0 & 39.7 \\
\hline
\end{tabular}


de riesgo de suicidio cuando la relación con la madre fue de mala a muy mala y $78 \%$ de los no casos cuando la relación fue buena o muy buena (especificidad $=63 \%$, sensibilidad $=73 \%$ ) (cuadro 4).

El modelo con la pareja generó tres segmentos finales: el primero predijo 91\% de las mujeres sin riesgo de suicidio cuando no habían recibido maltrato emocional de su pareja; en los otros dos segmentos se predijo, primero, $78 \%$ de los casos con riesgo suicida cuando recibieron maltrato emocional y físico de la pareja, y 57\% sin riesgo suicida cuando recibieron maltrato emocional, pero no recibieron maltrato físico (cuadro 4) (especificidad=97\%, sensibilidad=18\%).

En relación al consumo de alcohol el modelo generó tres segmentos finales. El primero predijo $67 \%$ de los casos con riesgo de suicidio cuando se habían desarrollado efectos perjudiciales en la salud. Los otros dos segmentos predijeron, primero, $63 \%$ de los casos de riesgo de suicidio cuando presentaron un efecto perjudicial en sus labores domésticas; en cambio, cuando no hubo este efecto, se predijo el $80 \%$ de no casos de riesgo de suicidio (especificidad $=78 \%$, sensibili$\mathrm{dad}=69 \%$ ) (cuadro 4).

El modelo con violencia sexual generó dos segmentos finales. El primero predijo $64 \%$ de los casos con riesgo de suicidio cuando las trabajadoras sexuales fueron violadas alguna vez; y el segundo segmento predijo $71 \%$ de los casos sin riesgo de suicidio cuando ellas no fueron violadas (especificidad $=84 \%$, sensibilidad $=45 \%$ ) (cuadro 4 ).

\section{DISCUSIÓN}

Los datos de este trabajo son relevantes por abordar problemas de salud mental que han sido poco investigados en poblaciones de difícil acceso, como la de trabajadoras sexuales.

Cuadro 4. Porcentajes de clasificación de casos y no casos de riesgo de suicidio en trabajadoras sexuales de Hidalgo $(n=103)$

\begin{tabular}{lccc}
\hline & \multicolumn{3}{c}{ Pronosticado } \\
\cline { 2 - 4 } & $\begin{array}{l}\text { Sin riesgo de } \\
\text { suicidio (\%) }\end{array}$ & $\begin{array}{c}\text { Riesgo de } \\
\text { suicidio (\%) }\end{array}$ & $\begin{array}{c}\text { Correcto } \\
(\%)\end{array}$ \\
\hline $\begin{array}{l}\text { Familia: Relación con la madre } \\
\text { - Sin riesgo suicida }\end{array}$ & 39.0 & 23.0 & 62.9 \\
- Riesgo suicida & 11.0 & 29.0 & 72.5 \\
$\begin{array}{l}\text { Pareja: Maltrato emocional } \\
\text { - Sin riesgo de suicidio }\end{array}$ & 60.0 & 2.0 & 96.8 \\
- Riesgo de suicidio & 33.0 & 7.0 & 17.5 \\
$\begin{array}{l}\text { Consumo de alcohol: Efectos } \\
\text { perjudiciales }\end{array}$ & & & \\
- Sin riesgo de suicidio & 49.0 & 14.0 & 77.8 \\
- Riesgo de suicidio & 12.0 & 27.0 & 69.2 \\
Violencia sexual: Violación & & & \\
- Sin RS & 53.0 & 10.0 & 84.1 \\
- RS & 22.0 & 18.0 & 45.0 \\
\hline
\end{tabular}

El estudio de la salud mental en este grupo se ha abordado de manera escasa en el mundo y en México es casi inexistente, destacando el de Ulibarri et al., ${ }^{14}$ realizado en 2010 en sexoservidoras de Tijuana y Ciudad Juárez, en el que describieron las características y la prevalencia del abuso sexual y físico, y su asociación con los síntomas de la depresión.

En el presente trabajo se exploraron los correlatos psicosociales relacionados con la depresión y con el riesgo de suicidio en una muestra de 103 trabajadoras sexuales del Estado de Hidalgo, México. La frecuencia de depresión fue de $39.8 \%$, la cual es muy superior a la reportada por la Encuesta de Epidemiología Psiquiátrica para la prevalencia de cualquier trastorno afectivo en las mujeres de población general $(3.0 \%)^{15}$ y a la global en la población general. ${ }^{16}$ La frecuencia de riesgo de suicidio -que incluye a la ideación, la planeación y al intento de suicidio- fue de 39\%, que también es más elevada que la de las mujeres de población general de nuestro país, donde la prevalencia de ideación suicida fue de $10.9 \%$, la de plan suicida fue de $1.5 \%$ y la de intento suicida fue de $1.0 \% .{ }^{17}$ Estos datos evidencian que las prevalencias no se distribuyen aleatoriamente en las poblaciones, sino que se concentran en las de mayor vulnerabilidad. ${ }^{18}$

Además de la magnitud de la frecuencia de depresión y de riesgo de suicidio, cabe notar que las mujeres de la muestra eran jóvenes adultas, con un promedio de 29 años, que requieren atención para su salud mental; la que si no se realiza de manera oportuna, tenderá a agravar los problemas y a afectar áreas importantes de convivencia como la familia y el entorno laboral. ${ }^{1}$

Las variables que predijeron los casos de depresión y los de riesgo de suicidio en el ámbito familiar fueron tener una mala o muy mala relación de la madre con la hija, en términos de falta de afecto, falta de comunicación e interés en las actividades e inquietudes de la hija, así como falta de supervisión y establecimiento de límites. Estos contenidos, traducidos en términos de las prácticas de crianza como rechazo emocional y negligencia, por sus consecuencias, se consideran formas de violencia familiar. ${ }^{19}$

Haber sufrido maltrato emocional por parte de la pareja también predijo la depresión y el riesgo de suicidio; por lo que es la violencia uno de los correlatos relacionados con ambos malestares en estas mujeres; la violencia perpetrada por la pareja es la que incide directamente en el estado depresivo y en la conducta suicida que se traduce en una forma de afrontamiento autodestructiva ante el sufrimiento derivado del maltrato.

El impacto de la violencia en la salud mental de las trabajadoras sexuales, y de las mujeres en general, está ampliamente documentado; en un estudio se observó que una de cada cinco mujeres que acudían a un centro de salud reportó haber experimentado algún tipo de violencia de parte de su pareja. ${ }^{20}$

El conglomerado de violencia en el ámbito familiar y de la pareja así como el antecedente de abuso sexual, el cual 
generalmente es ejecutado por familiares o personas conocidas, pone de manifiesto el alto costo que cobra sobre la salud mental de las mujeres a corto y a largo plazo.

La alta proporción de depresión y riesgo de suicidio en las trabajadoras sexuales de este estudio coinciden con las otras reportadas en otro estudio en trabajadoras sexuales de Tijuana que encontró que más del $80 \%$ presentó sintomatología depresiva elevada; ${ }^{14}$ y con otros estudios que han documentado frecuencias de problemas de salud mental mayores en trabajadoras sexuales que en las mujeres de la población general.,21

Respecto al riesgo de suicidio en particular, un predictor importante fue el relativo a las consecuencias negativas del consumo de alcohol sobre la salud física y el hogar. Una de las propuestas de la $\mathrm{OMS}^{22}$ se refiere a la responsabilidad compartida que tienen los productores de bebidas alcohólicas y los mediadores -como este caso que la actividad laboral preponderante es en bares-, además de los gobiernos y el sector salud para realizar estrategias enfocadas en la reducción del daño; es decir, reducción de las consecuencias nocivas del consumo de alcohol. Para lograr esto es necesario traducir los altos costos de salud a términos económicos para sensibilizar a las autoridades de los diversos sectores implicados, incluidos quienes propician el consumo de alcohol en la actividad laboral de las trabajadoras sexuales: "sólo la evidencia de este impacto económico negativo podría convencer a los sectores dirigentes del sector salud y de otros". ${ }^{23}$

Otro predictor para el riesgo de suicidio se refiere a la violencia sexual, específicamente a la violación. El haber sido víctima de violación hace referencia a una victimización física y emocionalmente significativa cuya consecuencia deriva en ideación, planeación y/o intento de suicidio como una forma autodestructiva de afrontamiento ante el sufrimiento derivado de la violación y de sus implicaciones morales y sociales. ${ }^{20}$

En su conjunto, los predictores de depresión y de riesgo de suicidio se identifican en el conglomerado de la violencia familiar, de pareja y laboral (ante la presión de beber) y los resultados coinciden con lo reportado en otro trabajo. ${ }^{10}$ Los predictores identificados evidencian la necesidad de atender estos problemas de salud mental como consecuencias de la victimización pasada y presente. En este sentido, es importante considerar los resultados obtenidos a fin de propiciar acciones para reducir o eliminar la violencia de pareja, fomentar la adquisición de habilidades para el manejo de consecuencias negativas del pasado e implementar estrategias para reducir el daño ocasionado por el consumo de alcohol, dadas las características del entorno laboral de las trabajadoras sexuales, ya que "el trabajo sexual es una profesión extremadamente peligrosa y el uso de estrategias de reducción del daño puede salvaguardar las vidas de las trabajadoras sexuales". ${ }^{24}$

Los resultados de este trabajo presentan un panorama de algunos malestares en la salud mental de un grupo po- blacional en situación de alta vulnerabilidad y contribuye como un antecedente que plantea cuestiones interesantes sobre el impacto que esto puede tener sobre la salud general y la vida de las trabajadoras sexuales, ${ }^{25}$ así como retos importantes para profundizar en la investigación de estos temas y el desarrollo de intervenciones. Sin embargo, también contiene aspectos que se podrían mejorar, como el trabajo con muestras más grandes y representativas; cabe recordar que cuando se trabaja con poblaciones de difícil acceso, como en este caso, existen circunstancias adyacentes que limitan el trabajo de recolección de datos, por ejemplo, los dueños de los establecimientos donde ellas ejercen, sus parejas o sus proxenetas limitan su tiempo y censuran sus actividades. Por ello, es importante plantear estudios considerando este elemento en el diseño y la planeación, además de otros estudios de seguimiento que permitan generar evidencia más concluyente sobre el efecto de las circunstancias a las que se enfrentan estas mujeres.

\section{AGRADECIMIENTOS}

A la psicóloga Edith Rivera Guevara como consultora estadística por su impecable asesoría; y al Programa de Igualdad entre Hombres y Mujeres por el financiamiento otorgado.

\section{REFERENCIAS}

1. Medina-Mora ME, Borges G, Lara C, Benjet C et al. Prevalencia de trastornos mentales y uso de servicios: resultados de la Encuesta Nacional de Epidemiología Psiquiátrica en México. Salud Mental 2003;26(4):1-16.

2. Benjet C, Borges G, Medina-Mora ME, Fleiz C et al. La depresión con inicio temprano: prevalencia, curso natural y latencia para buscar tratamiento. Salud Pública Mex 2004;46(5):417-424.

3. Opoliner A, Blacker D, Fitzmaurice G, Becker A. Challenges in assessing depressive symptoms in Fiji: A psychometric evaluation of the CES-D. Int J Soc Psychiatry 2013; publicado en línea. Consultado: marzo 2014.

4. INEGI. Datos de mortalidad general, por tipo de defunción. México: 2012.

5. Carroll PW, Berman AL, Maris RW, Moscicki EK et al. Beyond the Tower of Babel: A nomenclature for suicidology. Suicide Life Threat Behav 1996;26(3):237-252.

6. Maris R W. Suicide. Lancet 2002;360(9329):319-326.

7. Surratt H, Weaver J, Inciardi J. The connections of mental health problems, violent life experiences, and the social milieu of the "stroll" with the HIV risk behaviors of female sex workers. J Psychol Hum Sexuality 2005;17:23-44.

8. Gilchrist G, Gruer L, Atkinson J. Comparison of drug use and psychiatric morbidity between prostitute and non-prostitute female drug users in Glasgow, Scotland. Addict Behav 2005;30:1019-1023.

9. Potterat J, Brewer D, Muth S, Rothenberg R et al. Mortality in a longterm open cohort of prostitute women. Am J Epidemiol 2004;159(8):778785.

10. Hong Y, Li X, Fang X, Zhao R. Correlates of suicidal ieation and attempt among female sex workers in China. Health Care for Women International 2007;28:490-505.

11. Shahmanesh M, Wayal S, Cowan F, Mabey D et al. Suicidal behavior among female sex workers in Goa, India: The silent epidemic. Am J Public Health 2009;99(7):1239-1246. 
12. International Neuropsychiatric Interview. MINI. Versión en Español (Sudamérica y Centroamérica) 5.0.0. DSM-IV. 1998.

13. SPSS para Windows, versión 19.0. Illinois: SPSS INC; 2010.

14. Ulibarri M, Hiller P, Lozada R, Rangel G et al. Prevalence and characteristics of abuse experiences and depression symptoms among injection drug-using female sex workers in Mexico. J Environ Public Health 2013;60(4):367-376.

15. Medina-Mora ME, Borges G, Benjet C, Lara MC et al. Estudio de los trastornos mentales en México: Resultados de la Encuesta Mundial de Salud Mental. En: Rodríguez JJ, Kohn R, Aguilar-Gaxiola S (eds.). Epidemiología de los trastornos mentales en América Latina y el Caribe. Washington, D.C.: Organización Panamericana de la Salud; 2009.

16. Belló M, Puentes E, Medina-Mora ME, Lozano R. Prevalence and diagnosis of depression in Mexico. Salud Pública Mex 2005;47(1):4-11.

17. Borges G, Medina-Mora ME, Orozco R, Ouéda C et al. Distribución y determinantes sociodemográficos de la conducta suicida en México. Salud Mental 2009;32(5):413-425.

18. Kessler R, Aguilar-Gaxiola S, Alonso J, Chatterji S. Special articles. The global burden of mental disorders: An update from the WHO World Mental Health (WMH) surveys. Epidemiologia Psichiatria Sociale 2009;18(1):23-33.
19. Torío S, Peña JV, Rodríguez MC. Estilos educativos parentales. Revisión bibliográfica y reformulación teórica. Teor Educ 2008;20:151-178.

20. Ramos L, Saltijeral MT, Romero M, Caballero MA et al. Violencia sexual y problemas asociados en una muestra de usuarias de un centro de salud. Salud Pública Mex 2001;43(3):182-191.

21. Gu J, Lau JTF, Chen H, Tsui $\mathrm{H}$ et al. Prevalence and factors related to syringe sharing behaviours among female injecting drug users who are also sex workers in China. Int J Drug Policy 2011;22(1):26-33.

22. Organización Mundial de la Salud (OMS)-Organización Panamericana de la Salud (OPS). Guía internacional para vigilar el consumo de alcohol y sus consecuencias sanitarias, Washington, DC: 2000.

23. Menéndez EL, di Pardo RB. Alcoholismo: políticas e incongruencias del sector salud en México. Desacatos 2006;20:29-52.

24. Rekart ML. Sex-work harm reduction. Lancet 2006;366(9503):21232134.

25. Rodríguez E, Ramos L, Fuentes de Iturbe P, Morón A et al. Violencia y salud mental. Resultados de un estudio realizado en trabajadoras sexuales. En: Rodríguez E, Fuentes de Iturbe P (eds.). Visibilizando las necesidades de salud mental de las trabajadoras sexuales. México: Instituto Nacional de Psiquiatría; 2013.

Artículo sin conflicto de intereses 